\title{
Flattening the epidemic curve of COVID-19 in Sri Lanka: the public health response
}

\author{
- A Communiqué from the College of Community Physicians of Sri Lanka \\ Correspondence: kapjay613@gmail.com (iD https://orcid.org/0000-0001-9795-4342 \\ DOI: https://doi.org/10.4038/jccpsl.v26i1.8311
}

Received on: 30 March 2020

Accepted on: 31 March 2020

The College of Community Physicians of Sri Lanka (CCPSL) is the apex professional body for the practice of public health in Sri Lanka. The CCPSL functions as a strong and informed professional body to advocate promotion and prevention of disease of the people of Sri Lanka. The CCPSL is responsive to the current and emerging public health challenges conforms to evidence-based approaches and principles.

Countries can change the course of the pandemic, if the basic public health principles are adopted as guided by the WHO based on country-specific contexts. In this respect, the CCPSL strongly advocates that the current COVID-19 epidemic in Sri Lanka be controlled through a strong-willed, evidence-based public health response.

An epidemic curve is used to visualize and to predict when new cases are reported and at what speed, during a disease outbreak such as Covid-19. It depicts the number of cases by day, week, or month in the geographical location. Epidemics follow a plot line of increasing cases, and eventually resolve, whether responding to interventions or having exhausted the supply of susceptible persons.

An outbreak progresses through the following stages:

Stage 1: No cases - No reported cases

Stage 2: Sporadic cases - One or more cases, imported or locally acquired

Stage 3: Clusters of cases - Most cases of limited transmission linked to chains of transmission of either being exposed to a family member being exposed (family cluster) or to neighbours or other close contacts (village clusters)

Stage 4: Community transmission - Cases without an epidemiologic link are common in the community

Up to date, 571,678 confirmed cases and 26,494 deaths have been reported world-wide due to Covid-19 pandemic and in Sri Lanka, there are 117 confirmed cases with only one death. We are now in stage 3 of clustering of the cases within villages. Every effort should be made to prevent progression of the disease to next level, since it would be very difficult to stop the increase of cases with community transmission beyond the current stage that we are in. 
The response to any epidemic of this nature includes (Figure 1):

1. Totally containing the outbreak at a very early stage

2. Flattening the curve

3. Raising health system capacity

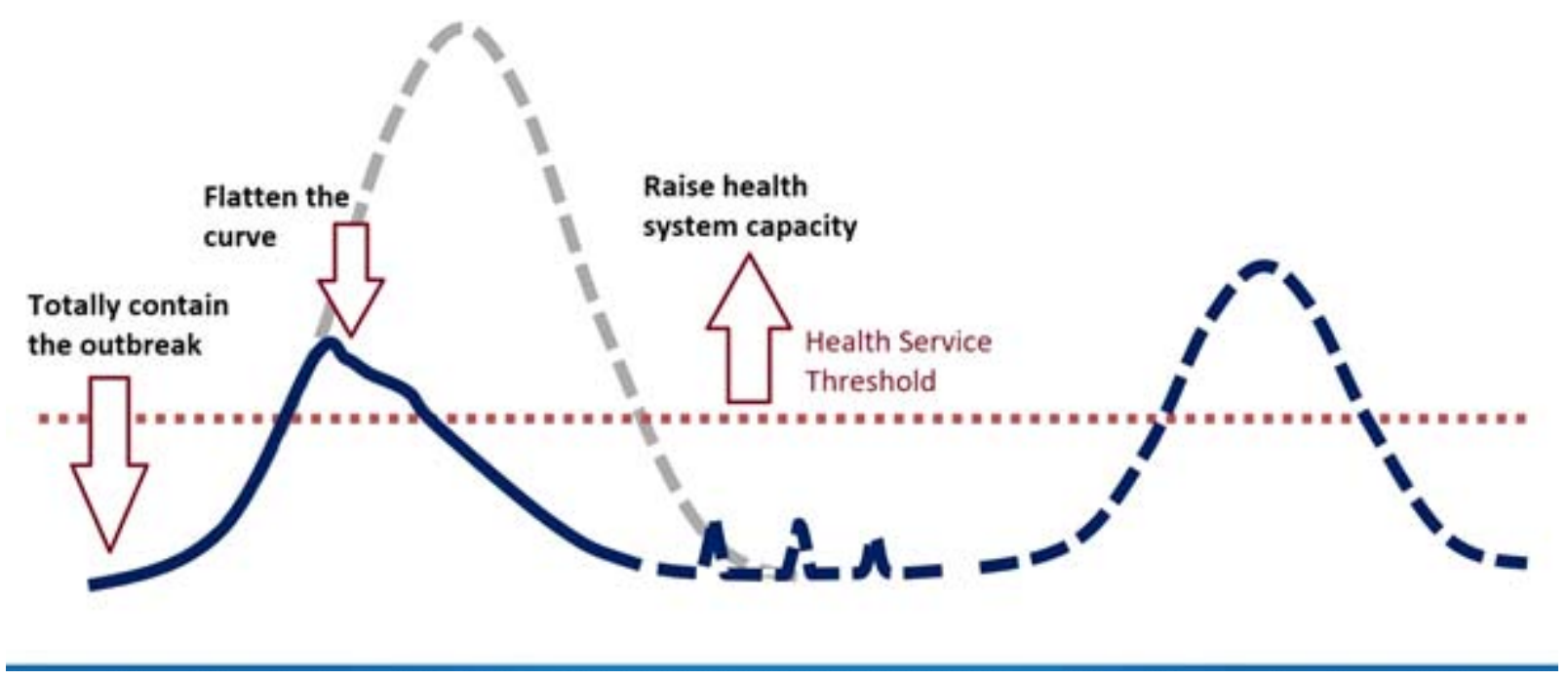

Figure 1. Epidemic response

'Containment' through a comprehensive approach (first response) would still be the major objective as the cases originating from local transmission are few. We also consider 'flattening the curve' (second response) may be the next best to slow the corona virus spread.

\section{What is flattening the curve?}

Flattening the curve means to keep the patient load at any given time at a level manageable by the health services. That is to slow down the transmission of the disease so that the health services will not be saturated. Usually, it takes approximately 2 weeks from the appearance of the first few cases to the onset of a larger outbreak. This interim period is the most crucial time for timely and appropriate public health measures.

\section{The country response}

In the absence of a COVID-19 vaccine, any country's aim has been to reduce contact rates in the population and thereby reduce transmission of the virus through non-pharmaceutical public health interventions. This could be achieved through two fundamental strategies:

- Suppression - aims to reverse the epidemic growth, reducing case numbers to low levels and maintaining that situation indefinitely

- Mitigation - focuses on slowing the epidemic spread -- reducing peak healthcare demand while protecting those most at risk of severe disease from infection 
Sri Lanka is mainly focusing on suppression of the curve, by applying a combination of public health interventions, such as social distancing of the entire population, home isolation of cases and household quarantine of their family, and school and university closures. Sequence of events is given below.

On $11^{\text {th }}$ March 2020, the first local case was identified in Sri Lanka. The reporting of a local case gave rise to the need for urgent and aggressive action and the health and non-health authorities executed a cascade of measures. The following figure depicts some of the key interventions implemented in Sri Lanka, in relation to the reported cumulative number of cases over the time (Figure 2).

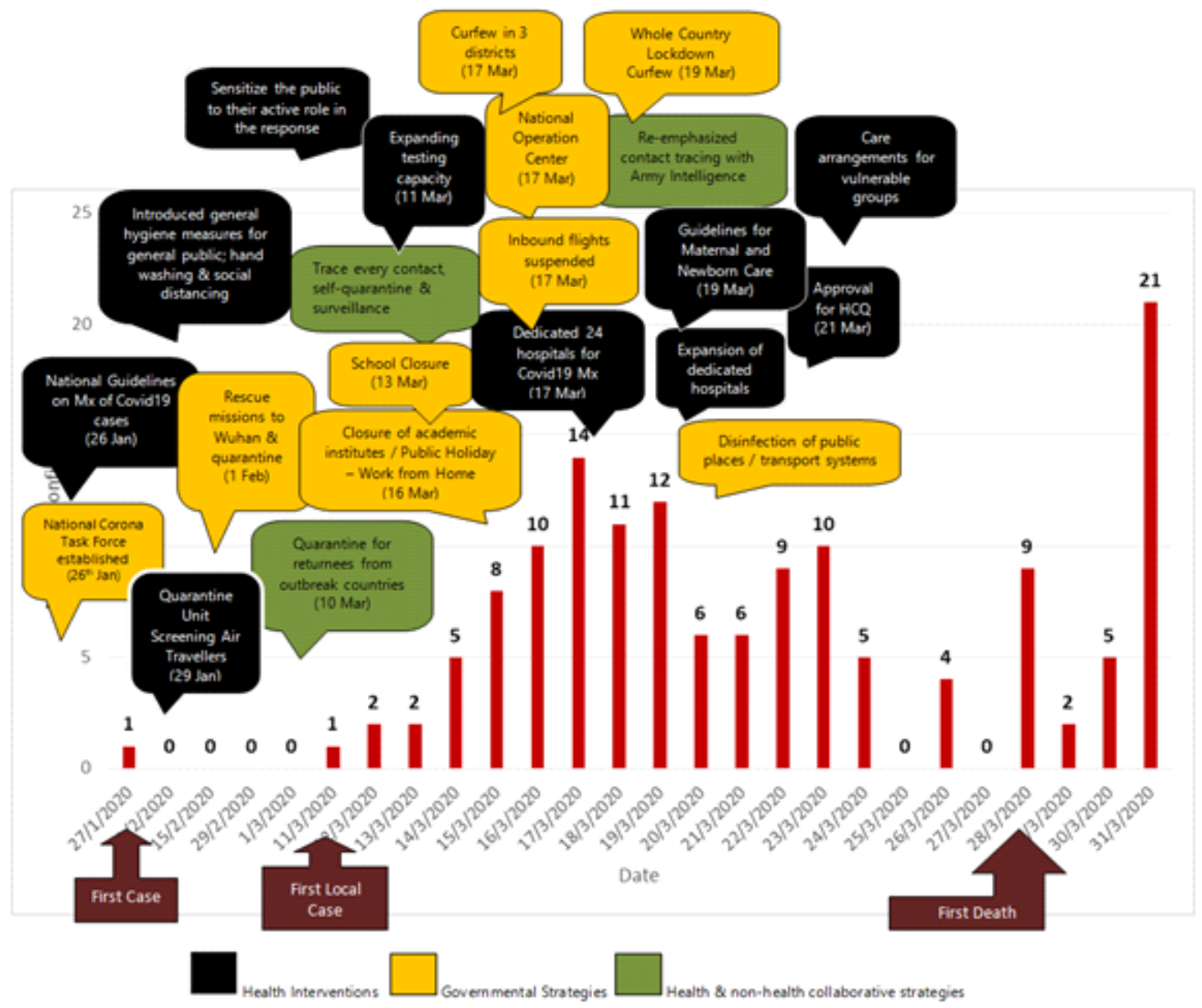

Figure 2. Cumulative number of cases and key interventions

Broadly, Sri Lanka implemented a whole-of-government, whole-of-society approach in the following key areas:

- Activate and scale up emergency response mechanisms

- Communicate with people about the risks and how they can protect themselves

- Find, isolate, test and treat every case and trace every contact

- Ready hospitals to protect and train health workers

- Encourage an all-of-society response and community spirit

- Implement all the non-health sector measures 
The country adopted timely and evidence-based approaches for the epidemic response advocated by the WHO:

1. Emergency response mechanisms

2. Risk communication and public engagement

3. Case finding, contact tracing and management

4. Surveillance

5. Public health measures (hand hygiene, respiratory etiquette and social distancing)

6. Laboratory testing

7. Case management

8. Infection prevention and control

9. Societal response (business continuity plans and whole-of-society approach)

\section{The role of public health hierarchy in this epidemic response}

The CCPSL places on record the active and substantial contribution of the entire public health hierarchy from the grass root level Public Health Inspector and Public Health Midwife to the highest central level public health policy makers and administrators in various capacities and facets of the epidemic response.

This emergency is declared by the WHO as a "Public Health Emergency". In response, under the directives of Hon. Minister of Health, Secretary, DGHS and Additional Secretary Public Health, the following preventive health teams have been in operation consistently:

- At national level - Deputy Director Generals Public Health, Directors in Public Health (Epidemiology Unit, Quarantine, Health Promotion Bureau, Disaster Management, Family Health Bureau), consultant community physicians, medical officers, special grade public health nursing officers and other staff supporting all the public health programmes are contributing at their highest capacity.

- At provincial and district level - The Provincial Director, Regional Director, consultant community physicians, medical officers, special grade public health nursing officer, special grade public health inspector and all Provincial and District level staff and at Divisional level the soldiers of public health led by the regional epidemiologists, medical officers of maternal and child health (MO-MCH), medical officers of health $(\mathrm{MOH})$, public health nursing sisters, public health inspectors, public health midwives all other categories work as team which has shown a remarkable sense of team.

The primary medical care teams also play a critical role in preventing and early detection efforts at the grassroot level including general practitioners.

\section{Public health teams contribute in the following areas:}

1. Epidemiology Unit - As the National Focal Centre of infectious diseases, the Epidemiology Unit advocates policy makers, formulate guidelines, execute a stringent surveillance on cases, contact tracing, collate data and disseminate information for the entire country. The highest-level technical guidance and advocacy on the epidemic response is originating from the Epidemiology Unit, and the success the country has achieved so far is largely due to the continued activities of the Epidemiology Unit guided by Chief Epidemiologist.

2. Quarantine Unit - It launched an early response, starting with active screening of all travellers coming into the country with thermal scanners at all points of entry to the country. Further, they referred all sick patients for medical care, educated all passengers on preventive measures, details retrieved from returnees from suspected countries were sent to respective $\mathrm{MOH}$ areas. They are currently working in collaboration with armed forces in the quarantine process for returning travellers. 
3. Health Promotion Bureau - It has implemented a pragmatic and successful media strategy using innovative modalities, and is acting as a reliable government body to take on the challenge of modifying behaviour and practices of people to face the epidemic, while counter-acting the misinformation and myths.

4. Family Health Bureau - It set out guidelines on pregnant mothers and children and closely coordinating with MOMCH, MOOH, PHNS, SPHM and PHM on the implementation of the activities at the ground level.

5. Ground Level - Early detection of cases and tracing close contacts are the evidence-based effective strategies during the early stage of an epidemic. A network of MOH staff with the guidance of Consultant Community Physicians and Regional Epidemiologists, nearly 900 medical officers of health from 357 $\mathrm{MOH}$ areas engage in contact tracing, surveillance on self-quarantined persons and educate general public with the active involvement of Public Health Inspectors and PHMs

6. Disaster Preparedness \& Response Division - This division is closely working with the public health teams

The caseload could have been many more if not the effective interventions initiated by public health teams in traveller screening, case detection, contact tracing, early reporting, early isolation/ quarantine and mass health education coupled with other strategies were in place. The public health efforts resulted in containing the epidemic without progressing into the stage of community transmission.

\section{Have we done enough - what has been shown to be effective?}

The epidemic is continuing. The country has implemented a multi-faceted, multi-stakeholder and societyoriented strategies at their best to counteract the expected damage. Learning from the successes and failures of other countries which faced similar situations would be of immense help to reshaping the current strategies and introducing new modalities.

We emphasize that "China has rolled out perhaps the most ambitious, agile and draconian disease containment effort in history”.

\section{China}

Sri Lanka

1. Unified and effective command system

2. Law- and science-based strategies

3. Coordinated deployment of resources

4. 4-Earlys (Early detection / Early Reporting / Early Isolation / Early Treatment) \& social mobilization

5. Rapid improvement in treatment capacity

6. Application of high tech

7. "For the people and of the people"

8. International exchange and cooperation
Early setting up the National Corona Task Force

WHO guided scientific strategies backed by Police and other armed forces by enforcing quarantine act

A National Operation Centre headed by Army Commander established. Sri Lanka Army, Police and other government agencies are deployed strategically All 4 Early's including prevention of imported cases done. Multi-sector social mobilization implemented

Expanding treatment facilities / ICU capacity. Mobilization of health staff

Digital health approach in case finding and contact tracing and media strategies Social mobilization with media Collective work with SAARC countries and WHO 
The following figures show interventions taken by different affected countries at different phases of the epidemic:

\section{Country}

South Korea

Japan

Singapore

Hong Kong

\section{Key strategy}

Early large-scale testing for Covid19 and trace contacts program

Strong social norms around obedience and mask wearing

Strict quarantine rules and contact tracing

School closures, quarantine and community response

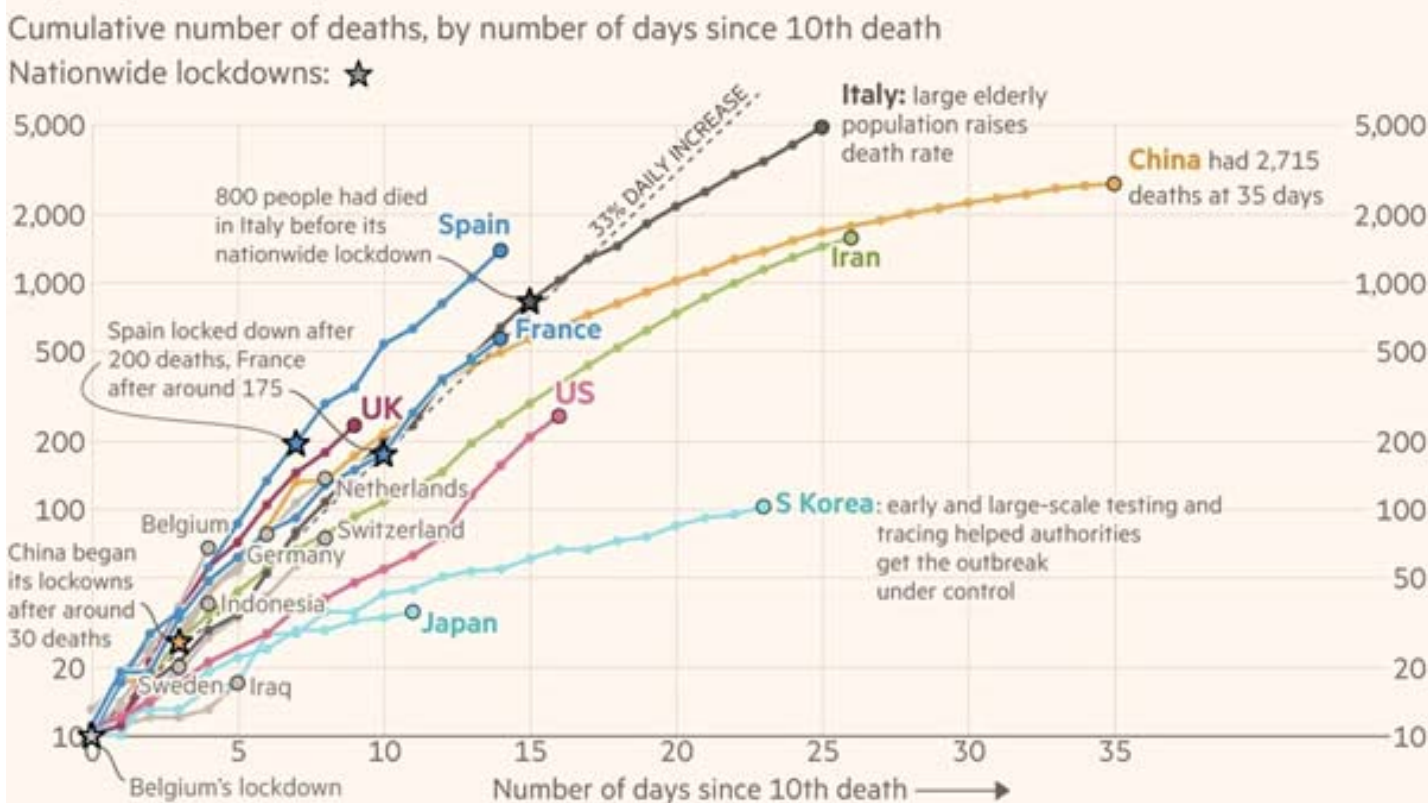

FT graphic: John Burn-Murdoch / @jburnmurdoch

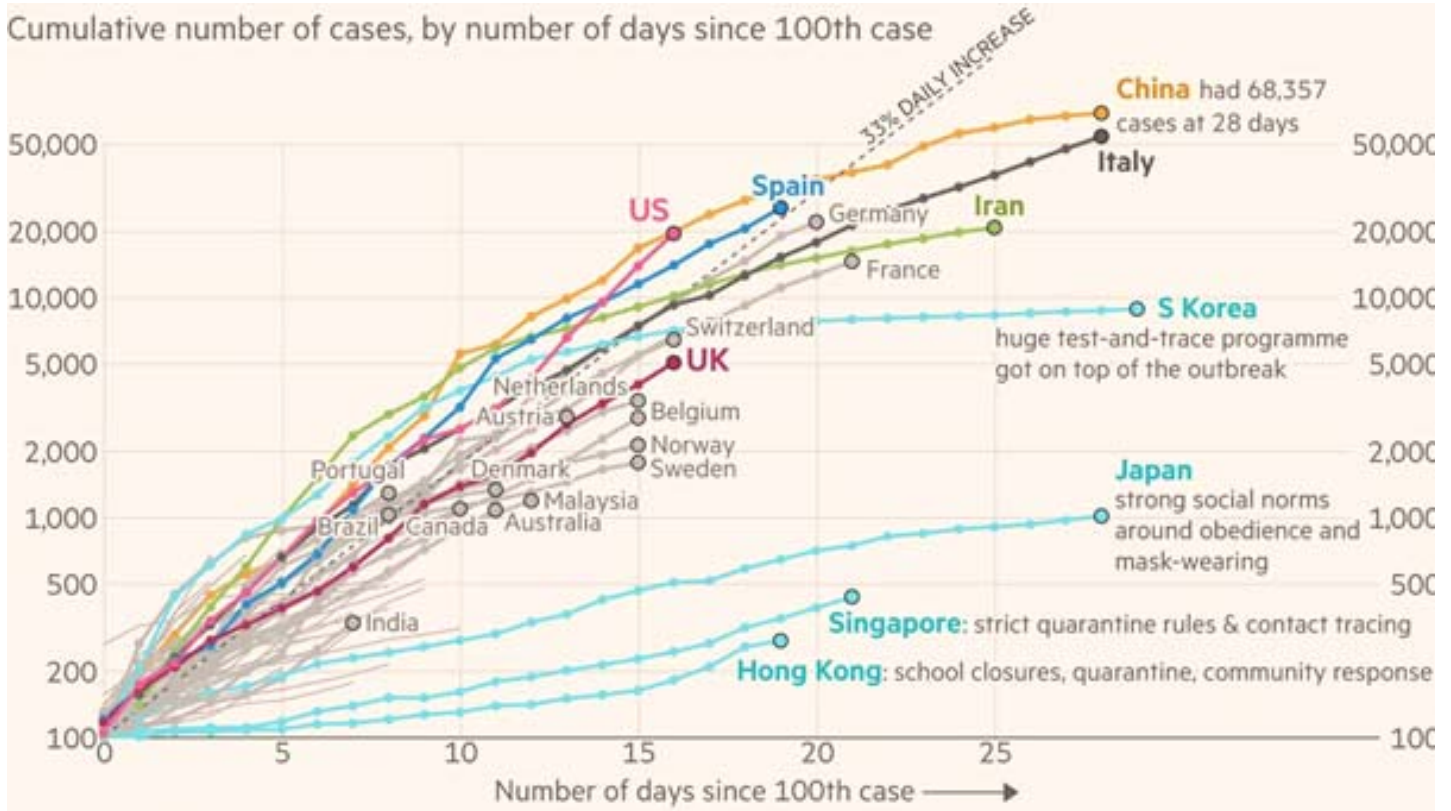

FT graphic: John Burn-Murdoch / @iburnmurdoch

Source: FT analysis of Johns Hopkins University, CSSE; Worldometers. Data updated March 21. 19.00 GMT 


\section{Are we on track?}

The CCPSL firmly believes that we are on the right track and that we have strategically implemented many of the interventions far ahead of other countries even without a single death being reported. However, the country needs to continue in the same path in heightened scale with appropriate new strategies. We acknowledge the strong political leadership provided by His Excellency President, Prime Minister and Minister of Health. Their commitment is in line with the Director General WHO's call for a "Whole Government Approach". The Army Commander's role and contribution brought success of the total quarantine process. This could not have been achieved with only the health sector. Sri Lanka Police is ensuring law and order during the social distancing. All the other ministries, agencies and professional bodies are supporting at their highest level in the response.

\section{The CCPSL proposals for the future challenges in the epidemic response}

Sri Lanka sets an example to the entire world, with its timely and effective response to the global epidemic despite being a low- and middle-income country. This highlights the importance of investing in public health. Although, we have done so far sensibly, the CCPSL proposes following strategies to address the future challenges in the epidemic response:

\section{Learn from the successes of other countries}

Success of China, South Korea and Japan and also the failures of Italy, Spain, Germany, UK and USA should be analyzed in depth and thereby, current strategies need to re-shaped. We should acknowledge the importance of;

- a whole government approach with optimal intersectoral collaboration and coordination

- a total system approach to manage health security (clinical services, population health, surveillance, science)

- transparency and accountability

2. Streamline the state of isolation or restricted access instituted as a strategy

Several issues have been encountered in the implementation of this strategy. To address the challenges, it is necessary to introduce a people-friendly intermittent relaxation of lockdown and improve social responsibility through culture change. It is imperative to strengthen non health measures such as availability of food and medicines, economic packages for all sectors affected, special care for financially vulnerable families, enforce laws to improve infection prevention and social distancing. We recommend that this strategy be reviewed after two weeks and change where necessary.

\section{Personal hygiene and social distancing}

Further strengthening community mobilization and mass campaign for personal hygiene and social distancing adopting new behaviours in the outbreak context should be considered as a 'nation at war against COVID -19 '. It is crucial that we sensitize the public to their active role in the response, such as on social and physical distancing - staying home and the one-meter rule at all times including transport.

\section{Sub-national and non-health sector preparedness and response plans}

Engage with key partners to update national and sub-national preparedness and response plans. Building on the existing strategic preparedness and response plan of COVID-19, exploration of possible collaboration options with the non-health sector should be undertaken.

\section{Raising health system capacity and readiness}

In addressing the challenges in raising health system capacity and readiness, the aims should be:

- preventing transmission in facilities and homes and not to overburden system 
- $\quad$ ensure capacity to treat severe and critical patients while maintaining essential health services

- protect health workers for continued care and service

When the severity profile of COVID-19 cases is considered; 40\% mild, 40\% moderate (with Pneumonia), $15 \%$ severe and only $5 \%$ become critical cases. Severe patients need oxygen therapy and critical cases need mechanical ventilation. Estimated numbers requiring hospitalization based on current size of outbreak cannot be precisely predicted. At the same time, it is essential to expand the capacity of mild patient admission and to deploy/ surge medical teams from nationwide depending on the increasing case load.

\section{Self-quarantine process}

More stringent quarantine process for persons exposed and close contacts should be deployed. Singapore showed the success of the strict quarantine rules. Introduce quarantine for persons with fever/other symptoms, suspects and close contacts for better compliance and outcomes.

\section{Protection and appreciation of key healthcare workers}

Sustenance of the epidemic response is largely dependent on the active involvement of the healthcare workers. It is equally important to protect them from being victims of the epidemic itself and also to keep their morale high. This is very much needed for public health staff as their contribution has not been adequately recognized or appreciated at national level. Ultimately, they might become unsung heroes of the ongoing battle. It is important to ensure adequate supply of personal protective equipment (PPE) for all the HCW dealing with patients and in preventive sector, and also to ensure the rational use of any PPE based on Ministry of Health guidelines which was adopted from WHO guidelines.

\section{Disease stigma}

The CCPSL is concerned with the stigma generated on identified cases and contacts by officials and media. We, CCPSL, reiterate that stigma is harmful to people and to the outbreak response. Stigma can drive people to hide the illness to avoid discrimination, stop people from seeking health care immediately and prevent people from adopting healthy behaviours.

\section{Sustaining care for the non-COVID patients}

Ensuring survival of the other patients is also crucial. When the whole system is geared to protect people from Covid-19, the focus for other disease entities and non-Covid patients will be diluted. Therefore, it is essential to introduce triage systems, reserving tertiary main hospitals for non-COVID patients. Vulnerable groups include: patients with chronic diseases who need regular treatment and drugs, e.g. dialysis; critical patients such as cancer patients, and those who have to seek rare medical treatment elsewhere; pregnant women who need regular prenatal check-ups; older people, those unable to leave the house and residents of different kind of institutions.

10. Further collaborations with media on providing public awareness on COVID and getting the public mobilized to meet their obligations as responsible citizens through dissemination of authentic and evidence-based messages and information on the epidemic. There is already a successful media campaign launched by the Health Promotion Bureau. It should be strengthened by giving dedicated media time for dissemination of current epidemic information.

11. Compilation of the evidence of COVID-19 of all countries in a dynamic e-repository.

\section{The future challenges}

Epidemic response is a dynamic process and strategies may also need to change depending on the dynamics of the outbreak. The general public needs to understand this reality and have a right to know the true facts. They should develop trust on the government's country-specific approaches and maximally support such efforts as all 
the interventions are executed with a valid reason and purpose. However, both the authorities and the people need to think carefully, weigh risks in context, and pursue interventions commensurate with the magnitude of the challenge.

We are reaping the gains again of the well-structured public health system of this country bringing about astounding results in this pandemic situation. In Sri Lanka, already existing public health system consisting of a battalion of field health staff are geared for needy interventions which is seen as a feasible task and an approach immensely helping to flatten the curve.

At the same time, it should not be ignored that we may be at greater risk of exaggerated fears and misplaced priorities as history suggests. We should understand the limited evidence on epidemic progression in tropical countries like Sri Lanka. With 95 years of experience in having a strong public health system and being a tropical country; the behaviour of the epidemic will be more likely to be different to the temperate countries. As such, what may be predicted may not be applicable in unique contexts of our country.

We urge the government, community leaders, other stakeholders and general public on the need of concerted efforts to fight against the country's worst challenge in the millennium.

At the same time, the CCPSL is happy that the Covid-19 outbreak forced an entire community to take refuge in multiple healthy behaviours which would have never been adopted with the traditional health education approaches and behaviour change. Capitalizing on the context of the Covid-19 outbreak, our long-term target is a "Healthy Sri Lanka".

\section{College of Community Physicians of Sri Lanka}

\section{March 2020}

